\title{
Posibilidades y limitaciones del Trayecto de la Práctica en tiempos de Pandemia. El caso del Profesorado en Matemática de la UNR
}

\author{
Possibilities and limitations of the Practice Path in times of \\ Pandemic. The case of the UNR Faculty in Mathematics
}

Sgreccia Natalia

https://orcid.org/0000-0003-2988-7410

sgreccia@fceia.unr.edu.ar

Facultad de Ciencias Exactas, Ingenieria y Agrimensura

| Universidad Nacional de Rosario | Argentina
Cirelli Mariela

https://orcid.org/0000-0002-5805-7515

cirelli@fceia.unr.edu.ar

Facultad de Ciencias Exactas, Ingenieria y Agrimensura

| Universidad Nacional de Rosario | Argentina

\section{RESUMEN}

Este trabajo se ubica en el desarrollo del trayecto de la práctica docente en el contexto de pandemia del año 2020, tomando como caso el Profesorado en Matemática de la Universidad Nacional de Rosario. Esta Universidad, así como otras en el territorio nacional, ha optado por aproximarse a una pedagogía de la virtualización durante el período de aislamiento. Para la carrera en cuestión, el trayecto de la práctica docente se constituye en su proyecto articulador, en tanto se desarrolla intencional e integralmente del primer al cuarto y último año del plan de estudios. En este marco, el presente trabajo retoma testimonios de docentes de los espacios curriculares respectivos. Puntualmente las profesoras comparten pareceres y experiencias relativos tanto a las similitudes como a las diferencias con respecto al aula presencial en términos sociales, tecnológicos, institucionales y de contenido. También mencionan dificultades que están teniendo en este contexto y señalan, a su vez, aspectos que podrían estar potenciándose en comparación con lo que suele suceder en el aula presencial. Finalmente identifican limitaciones propias de la virtualidad para el desarrollo del trayecto de interés, sin dejar de apuntar posibilidades que en estas circunstancias están emergiendo. Estas reflexiones situadas permiten robustecer el conocimiento acerca de las adaptaciones de los dispositivos de formación para ser profesor/a en Matemática que se emplean en la carrera. Una comprensión del asunto desde la investigación educativa posibilita no solo responder a la coyuntura actual, sino seguir mejorando los mecanismos para contribuir a la sociedad con profesionales idóneos/as, con herramientas para descifrar las necesidades de su tiempo, en clave de compromiso social universitario.

\section{PALABRAS CLAVE}

Profesorado en

Matemática,

Práctica Profesional

Docente,

Virtualización,

Pandemia. 
KEY WORDS

Teaching staff in Mathematics, Professional Teaching Practice,

Virtualization

Pandemic.

\section{ABSTRACT}

This paper is located in the development of the teaching practice path in the context of the pandemic of the year 2020, taking as a case the Faculty in Mathematics of the National University of Rosario. This university, as well as others in the national territory, has chosen to approach a pedagogy of virtualization during the period of isolation. For the career in question, the path of teaching practice constitutes its articulating project, as it is developed intentionally and comprehensively from the first to the fourth and last year of the study plan. In this framework, the present work takes up testimonies of teachers from the respective curricular spaces. Specifically, the teachers share opinions and experiences regarding both the similarities and the differences with respect to the classroom in social, technological, institutional and content terms. They also mention difficulties they are having in this context and, in turn, point out aspects that could be enhancing themselves compared to what usually happens in the classroom. Finally, they identify limitations inherent in virtuality for the development of the path of interest, without ceasing to point out possibilities that in these circumstances are emerging. These situated reflections allow to strengthen the knowledge about the adaptations of the training devices to be a teacher in Mathematics that are used in the career. An understanding of the matter from educational research makes it possible not only to respond to the current situation, but also to continue improving the mechanisms to contribute to society with qualified professionals, with tools to decipher the needs of their time, in the key of university social commitment. 


\section{PRESENTACIÓN DEL CONTEXTO}

La carrera Profesorado en Matemática de la Universidad Nacional de Rosario (UNR) está radicada en el Departamento de Matemática de la Escuela de Ciencias Exactas y Naturales de la Facultad de Ciencias Exactas, Ingeniería y Agrimensura. Con la premisa de ofrecer profesionales de la Educación Matemática con una sólida formación en la región de influencia (sur de la provincia de Santa Fe), esta carrera fue creada en 1988, valiéndose de la Licenciatura en Matemática que existía desde un poco más de 20 años y del área de formación docente ofrecida por la Facultad de Humanidades y Artes de la misma Universidad. A más de 30 años de aquel momento, es preciso resumir que la carrera fue transformando su currículum plasmándose en dos cambios de plan de estudios (2002 y 2018), los espacios curriculares específicos de la carrera fueron configurando su propia identidad gradualmente y desde el año 2013 la totalidad de la carrera se encuentra funcionando íntegramente en el Departamento de procedencia.

De acuerdo al perfil del egresado/a proyectado desde el plan de estudios, el/la profesor/a en Matemática es un/a graduado/a universitario/a con una sólida formación matemática que a su vez integra saberes y procedimientos de otras áreas necesarios para el desarrollo de su trabajo disciplinar específico, y que los articula a partir de conocimientos teóricos y prácticos del campo educativo, para construir procesos de enseñanza-aprendizaje-evaluación desde una perspectiva social, política y cultural.

Acorde con la Res. CIN 856/13 (Consejo Interuniversitario Nacional, 2013), la propuesta curricular del Profesorado en Matemática de la UNR comprende cuatro campos de formación, con una distribución ponderada acorde a través del plan de estudios: disciplinar específica, pedagógica, general y de la práctica profesional docente (PPD). Puntualmente este último está dirigido a la articulación teórico-práctica de los demás campos de formación, integrándolos mediante actividades de diversa naturaleza con el objetivo de desarrollar competen- 
cias en el diseño, implementación, análisis y evaluación de prácticas educativas transformadoras en el área de la Matemática. Conformado por cuatro espacios curriculares anuales, se constituye en el proyecto articulador a lo largo de toda la carrera, en que cada año comprende sucesivas instancias de desmenuzamiento de la Matemática escolar así como trabajo en terreno en ámbitos educativos que se van intensificando y profundizando a través de la formación.

En particular, desde el Proyecto de Investigación "El trayecto de la Práctica Profesional Docente en el Profesorado en Matemática. El caso de la UNR" (1ING576, 2018-2021) se presta especial atención a los dispositivos de formación desplegados desde el aula de formación en el trayecto en cuestión. Cabe señalar que, en este marco, se conceptualiza a los dispositivos de formación en sintonía con lo puntualizado por Sanjurjo (2009) luego de un recorrido por varios referentes: aquellos artificios complejos, pensados y/o utilizados para plantear alternativas de acción, que se crean o se aprovechan para resolver problemáticas contextualmente, con un alto grado de maleabilidad para adecuarlos al análisis sobre las prácticas docentes.

Adicionalmente cabe señalar que el cursado del trayecto en su conjunto se desarrolla mediante la modalidad de taller, entendido como un formato orientado a la producción y quehacer requerido en la práctica profesional de un/a profesor/a en Matemática que propende a desarrollar alternativas de acción, a la toma de decisiones y a la producción de soluciones innovadoras para encarar los desafíos de la práctica. Se evalúa mediante trabajos prácticos, presentaciones orales y/o monografías que los/as estudiantes van concretando durante el cursado.

En esta ocasión, ubicados en la coyuntura actual de aislamiento físico preventivo dada la situación de pandemia a nivel mundial, se comparten pareceres de docentes del trayecto de interés acerca del desarrollo de las actividades curriculares en este contexto donde se propició la emergencia de una pedagogía de la virtualización (Copertari y Trottini, 2013). Se considera que una comprensión del asunto desde aproximaciones que puedan realizarse en términos de investigación educativa posibilita no solo responder a la coyuntura actual, sino seguir mejorando los dispositivos desplegados para contribuir a la sociedad cambiante del siglo XXI con profesionales idóneos/as, que dispongan de herramientas para descifrar las necesidades de su tiempo, en clave de compromiso social universitario (Gerlero, 2014).

\section{RELEVAMIENTO EFECTUADO}

Luego de sucesivas conversaciones informales entre las profesoras del trayecto de la práctica, en el marco del Proyecto 1 ING576 y con el objetivo de sistematizar experiencias, a un mes de iniciada la cuarentena se optó por aplicar un cuestionario abierto mediante Google Form a las docentes de los espacios curriculares en cuestión que no son autoras de este trabajo. 
Luego de sucesivas conversaciones informales entre las profesoras del trayecto de la práctica, en el marco del Proyecto 1 ING576 y con el objetivo de sistematizar experiencias, a un mes de iniciada la cuarentena se optó por aplicar un cuestionario abierto mediante Google Form a las docentes de los espacios curriculares en cuestión que no son autoras de este trabajo.

Se indagó, básicamente, sobre las siguientes cuestiones (remarcándose para esta ocasión los asuntos de interés en negrita):

- Señalar las principales similitudes con respecto al aula presencial que reconocen en términos sociales, tecnológicos, institucionales y de contenido.

- Análogamente, indicar las principales diferencias.

- Mencionar dificultades que se están teniendo en este contexto.

- Reconocer aspectos que se están potenciando, con respecto a lo que suele suceder en el aula presencial.

- Identificar limitaciones propias de la virtualidad para el desarrollo del trayecto.

- Análogamente, para las posibilidades que emergen.

- Otras consideraciones que le parecen relevantes compartir y no han sido contempladas previamente.

El plantel docente del campo de la PPD está constituido por ocho cargos (cuatro de profesor y cuatro de auxiliar), ocupados por seis personas (dos de ellas se desempeñan en dos espacios), de las cuales dos son las autoras del trabajo. Es así que se envió el cuestionario a cuatro personas, contando con la respuesta de tres ellas que, en conjunto, representan a la totalidad de las asignaturas.

- P1 se desempeña como auxiliar de PPD I y profesora de Residencia.

- P2 se desempeña como profesora de PPD II.

- P3 se desempeña como auxiliar tanto de PPD III como de Residencia.

Se transcriben sus respuestas a cada uno de los asuntos de interés y se realizan interpretaciones a partir del análisis de su contenido (Ander-Egg, 2003). 


\section{TESTIMONIOS DE LAS PROFESORAS}

Entre las principales similitudes con respecto al aula presencial (Tabla 1) reconocidas por las docentes, en términos sociales, tecnológicos, institucionales y de contenido, es posible advertir:

- Intercambio con los/as estudiantes, posibilitado a través de los recursos del aula virtual

- Abordaje del contenido, con adaptaciones de consignas

- Contacto constante, aunque no físico

- Organización de la actividad curricular, dado que se venía usando la plataforma

Tabla 1| Principales similitudes con respecto al aula presencial

\begin{tabular}{|c|c|}
\hline $\mathbf{P}$ & \multicolumn{1}{c|}{ Respuestas } \\
\hline $\mathbf{P 1}$ & $\begin{array}{l}\text { En cuanto a similitudes con el aula presencial destaco la posibilidad de intercambio con los estudiantes } \\
\text { a través de los distintos recursos que habilita el aula virtual (foros, archivos, tareas, entre otras), todo ello } \\
\text { con un marco de institucionalidad (pues las comunicaciones se realizan en el espacio institucional crea- } \\
\text { do para ello). El abordaje del contenido es igualmente posible, tal vez adaptando algunas consignas de } \\
\text { trabajo para aportar riqueza en los intercambios y devoluciones. En términos sociales, la imposibilidad de } \\
\text { "vernos" puede ser una desventaja. Si bien uno permanece en contacto constante, el decir con la mirada } \\
\text { con los gestos, con la postura, no es visible. }\end{array}$ \\
\hline P2 & $\begin{array}{l}\text { En términos sociales no se reconocen similitudes. En cuanto a lo tecnológico, se continúa de manera } \\
\text { similar a la presencial en cuanto a la entrega de trabajos relativos a lecturas teóricas. En cuanto a los con- } \\
\text { tenidos, se han cubierto todos los teóricos de un modo similar al aula presencial. }\end{array}$ \\
\hline P3 & $\begin{array}{l}\text { Considero que en cuanto a lo organizativo de la materia, no hay cambios significativos. Desde hace } \\
\text { algunos años ya venimos implementando el uso de espacios virtuales generados por la misma Facultad, } \\
\text { utilizado como repositorio de materiales, organizador de las distintas partes que conforman la materia y } \\
\text { las actividades de cada clase, lugar de entregas de esas actividades y es uno de los canales de comunica- } \\
\text { ción con los estudiantes. }\end{array}$ \\
\hline
\end{tabular}
Estas similitudes denotan que el funcionamiento troncal
del trayecto ha podido continuar, viéndose fortalecido por
la experiencia previa en cuanto al empleo de plataformas
educativas que ha sido robustecida, incorporando más fun-
cionalidades, a partir de la virtualización masiva producida.

Las principales diferencias con respecto al aula presencial (Tabla 2) que las profesoras puntualizan pueden resumirse en el aquí y ahora de la frescura de la clase en vivo y en directo (Sanjurjo, 2005), conjugando con componentes afectivos y anímicos que suelen salir a la luz en este tipo de espacios: 
- Imposibilidad de encuentros presenciales, a los que se aproximan con videollamadas

- Expresiones emocionales emergentes, que se perciben más fácilmente en la presencialidad

- Espontaneidad e interacción, en la tríada docente-estudiante-con tenido

Tabla 2 | Principales diferencias con respecto al aula presencial

\begin{tabular}{|c|c|}
\hline $\mathbf{P}$ & \multicolumn{1}{c|}{ Respuestas } \\
\hline P1 & $\begin{array}{l}\text { La principal diferencia radica en la imposibilidad de vernos. Asimismo, hemos implementado herramientas } \\
\text { de encuentro virtual (al estilo de videollamadas), por fuera del campus institucional, para lograr mayor acer- } \\
\text { camiento. Tal vez esa imposibilidad de vernos limita las expresiones emocionales que son muy propias de los } \\
\text { espacios de práctica. Aun así, podemos captar, en las expresiones de los estudiantes, cierta incertidumbre y } \\
\text { ansiedad en relación con el comienzo de sus prácticas (específicamente en la asignatura Residencia) y tam- } \\
\text { bién cierto desánimo en cuanto a las dificultades que les significa trabajar en equipo casi sin conocerse (en el } \\
\text { espacio de práctica de primer año del PM). }\end{array}$ \\
\hline P2 & $\begin{array}{l}\text { La socialización y los debates se implementan a través de foros, lo que implica un uso más intensivo del } \\
\text { mente no se utilizaban. La comunicación con estudiantes es asincrónica. El trabajo al interior de la cátedra } \\
\text { es un tanto errático, sin horarios establecidos. Hemos tenido que extender los tiempos de entrega de tra- } \\
\text { bajos prácticos por parte de estudiantes así como también la elaboración de las devoluciones respectivas } \\
\text { por parte del equipo de cátedra. }\end{array}$ \\
\hline P3 & $\begin{array}{l}\text { La presencialidad brinda una espontaneidad y una interacción en el corto plazo no solo entre los estu- } \\
\text { diantes, sino con los docentes y con el contenido. }\end{array}$ \\
\hline
\end{tabular}

Acerca de las dificultades que se están teniendo en este contexto (Tabla 3), las docentes destacan varias, distinguiéndose a su vez en qué tramo del trayecto se está:

- Inseguridades o limitaciones en el uso de herramientas tecnológicas

- Gestión del tiempo en producciones virtuales

- Organización de los tiempos de trabajo

- Cambios con respecto a la implementación de prácticas residentes en entornos virtuales

- Implementación de simulaciones de clases o microclases

- Conocimiento docente de los/as estudiantes produciendo en la presencialidad

- Funcionamiento de los grupos de estudiantes cuando no se conocen o algunos se desfasan

- Formatos para las retroalimentaciones

- Disponibilidad de libros de texto para las actividades

- Cantidad de estudiantes

- Sobrecarga laboral en docentes

- Incertidumbre con respecto a los nuevos escenarios 
Tabla 3 | Dificultades que se están teniendo en este contexto

\begin{tabular}{|c|c|}
\hline $\mathbf{P}$ & Respuestas \\
\hline P1 & 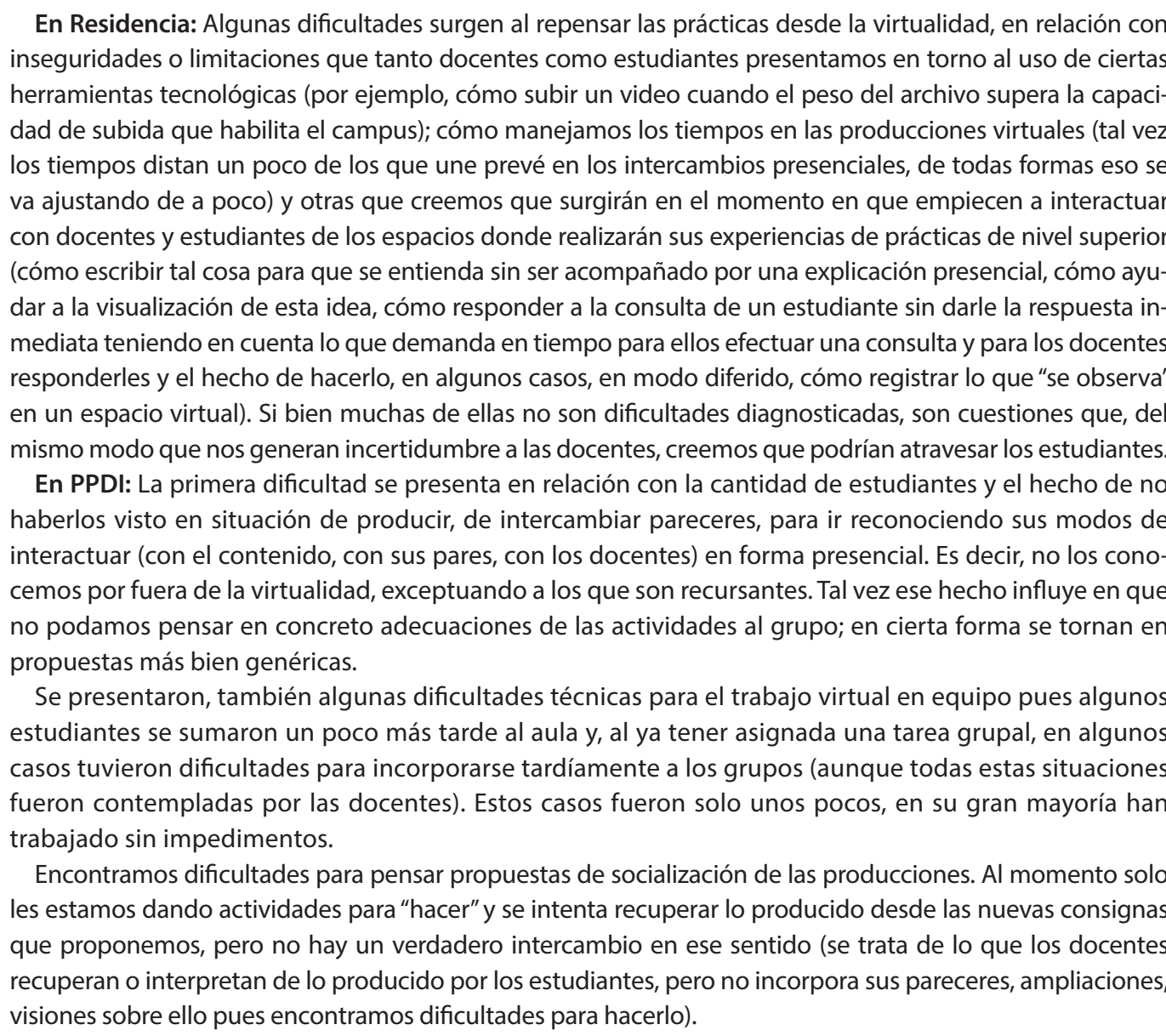 \\
\hline P2 & $\begin{array}{l}\text { Nada puede reemplazar el ambiente que se respira en una clase presencial. Personalmente tengo la sen- } \\
\text { sación que falta algo y que ese algo es muy importante. Algunos grupos de trabajo han tenido dificultades } \\
\text { cuando la consigna involucra búsqueda o análisis de actividades en libros de texto para estudiantes de } \\
\text { nivel secundario. Los/as estudiantes no disponen de ellos y no está habilitada la biblioteca. Se les han acer- } \\
\text { cado algunos archivos con partes de libros seleccionados y escaneados por la responsable de la cátedra asi } \\
\text { como también materiales virtuales, pero estos son escasos y al parecer resultan poco prácticos para los/ } \\
\text { as estudiantes. En este primer cuatrimestre, la mayor dificultad se presenta en la actividad de simulación } \\
\text { de clases. Si bien se podría pedir que graben videos, no nos parece pertinente debido a las herramientas } \\
\text { diferentes con las que puede contar cada estudiante, además, al ser grupal, se complicaría la coordinación } \\
\text { La planificación y simulación individual por video también fue descartada; un motivo es el mismo que se } \\
\text { expuso anteriormente para la implementación grupal y otro es que al ser } 13 \text { estudiantes demandaría un } \\
\text { tiempo adicional con el cual las docentes de la cátedra no contamos; estamos desbordadas con el trabajo } \\
\text { que implica la atención no presencial de clases en nuestras respectivas escuelas secundarias y con la ela- } \\
\text { boración de informes que nos solicitan desde los equipos directivos de las mismas. Esto último y lo que } \\
\text { sigue tiene relación con lo mencionado antes acerca de los horarios de trabajo erráticos. Creo que no se } \\
\text { le puede exigir nada a nadie (estudiantes o docentes) en un contexto de incertidumbre en el cual se han } \\
\text { alterado los estados emocionales y la vida extra e intra-familiar. }\end{array}$ \\
\hline P3 & $\begin{array}{l}\text { Considero que esas principales diferencias, pueden salvarse al menos en parte por el hecho de que la } \\
\text { presencialidad no era la base absoluta de las clases. Por lo que no considero que haya dificultades que no } \\
\text { puedan sobrellevarse de otra forma, al menos en estas primeras instancias. }\end{array}$ \\
\hline
\end{tabular}


De este modo, la combinación de los dominios disciplinar-pedagógico-tecnológico señalados por Mishra y Koehler (2006) entra en tensión en esta coyuntura, que puede constituirse al mismo tiempo en una oportunidad. Habrá que evaluarlo... Una opción podría haber sido "en este contexto el trayecto de la práctica se suspende en el año en curso", pero se fue hacia posibilidades de trabajo alternativas que pudieran no solo sobrellevar el momento sino fortalecer la formación de los/as futuros/as profesores en Matemática, ya entrando en la tercera década del siglo XXI. También interpela la apreciación de P3 en cuanto a cuán prescindible es la presencialidad en las clases que habitualmente se llevan a cabo. Pareciera insinuar que mientras menos imprescindible sea, menos dificultosa será la transición hacia la virtualidad.

\section{Una opción podría haber sido "en este contexto el trayecto de la práctica se suspende en el año en curso", pero se fue hacia posibilidades de trabajo alternativas que pudieran no solo sobrellevar el momento sino fortalecer la formación de los/ as futuros/as profesores en Matemática}

Al referirse a los aspectos que se están potenciando (Tabla 4), con respecto a lo que suele suceder en el aula presencial, indican:

- Escritura espontánea, en foros sincronizados

- Paciencia y compromiso, tanto de docentes como de estudiantes

- Herramientas virtuales de trabajo colaborativo, como parte básica del trayecto globalmente

- Seguimientos más personalizados, dado que en la oralidad ocurren más rápido

- Pausas en los debates, que favorecen la profundidad en el tratamiento

- Mejor reflexión de lo que se está trabajando, al extenderse los tiem-

pos de las clases

Tabla 4 | Aspectos que se están potenciando

\begin{tabular}{|c|c|}
\hline $\mathbf{P}$ & \multicolumn{1}{c|}{ Respuestas } \\
\hline \multirow{1}{*}{$\mathbf{P 1}$} & $\begin{array}{l}\text { En Residencia: Se potencia la escritura espontánea, al proponer intercambios a través de foros en momen- } \\
\text { tos puntuales. Se potencia la paciencia y el compromiso en muchos aspectos: los estudiantes son pacientes } \\
\text { con los tiempos de los docentes en relación con la redefinición de consignas, a la vez que los docentes flexi- } \\
\text { bilizan plazos y formatos de entrega (pues se reconoce que ciertas tareas, por ejemplo, toman más tiempo de } \\
\text { lo previsto), sin que esto signifique un desentendimiento de algunas de las partes, sino por el contrario en el } \\
\text { sentido de redefinir tiempos al reconocernos interactuando en espacios que, si bien ya veníamos utilizando, } \\
\text { lo hacíamos en forma complementaria con la presencialidad. Se reconoce un gran compromiso de ambas } \\
\text { partes para lograr que esta modalidad de trabajo funcione. } \\
\text { En PPDI: Se potencia el uso de herramientas virtuales de trabajo colaborativo (desde el campus se habili- } \\
\text { tan, por ejemplo, wikis, aunque no se descartan los intercambios al interior de subgrupos por otros medios } \\
\text { que puedan resultarles funcionales), cuestión que sienta las bases para esta modalidad de trabajo que es } \\
\text { central para los espacios de PPD. }\end{array}$ \\
\hline
\end{tabular}




\begin{tabular}{|c|c|}
\hline $\mathbf{P}$ & \multicolumn{1}{c|}{ Respuestas } \\
\hline $\mathbf{P 2}$ & $\begin{array}{l}\text { Los foros de socialización/debate permiten un seguimiento más individualizado de los aprendizajes en } \\
\text { virtud de los aportes que se realizan, así como también un rico intercambio entre pares, que en modalidad } \\
\text { presencial generalmente no se da, pues en la oralidad todo ocurre más rápido. El hecho de tener que leer } \\
\text { las intervenciones de sus pares o docentes para analizarlas y luego hacer aportes en base allas pone } \\
\text { pausa a los debates lo cual permite aparentemente reflexiones más profundas, más pensadas, incluso } \\
\text { con el debido respaldo teórico, que es alentado por la cátedra mediante interrogantes que promueven la } \\
\text { revisión de creencias y la conexión tanto con saberes previos como con los recientemente construidos o } \\
\text { en proceso de construcción. }\end{array}$ \\
\hline P3 & $\begin{array}{l}\text { Creo que ahora los tiempos de la clase son más extendidos, lo que puede favorecer a una mejor reflexión } \\
\text { de lo que se está trabajando. }\end{array}$ \\
\hline
\end{tabular}

Se coincide con lo reportado por Kemelmajer (2020) en cuanto a que esta situación está interpelando a educadores e instituciones educativas acerca de su capacidad para aprovechar las tecnologías y beneficiarse pedagógicamente mediante la digitalización y virtualización.

\title{
También señalan limitaciones propias de la virtualidad para el desarrollo del trayecto (Tabla 5) tales como:
}

\author{
- Encuentro presencial con otras personas en el contexto de la práctica \\ - Vivenciar un salón de clase mientras ensaya a ser profesor/a en ma \\ temática \\ - Vivenciar simulaciones de clases o microclases \\ - Sensaciones vividas en el intercambio diario \\ - Amplitud de libros de texto a disposición \\ - Trabajo en terreno en instituciones asociadas
}

Tabla 5 | Limitaciones propias de la virtualidad para el desarrollo del trayecto

\begin{tabular}{|c|c|}
\hline $\mathbf{P}$ & \multicolumn{1}{c|}{ Respuestas } \\
\hline P1 & $\begin{array}{c}\text { En Residencia: En el contexto de una práctica, la imposibilidad de vivenciar en primera persona el en- } \\
\text { cuentro presencial con el otro, tanto con el docente coformador como con los estudiantes, de experimen- } \\
\text { tar las sensaciones que genera el pararse frente al pizarrón en un aula repleta de alumnos, o el ponerse } \\
\text { en el lugar del estudiante que pregunta de manera espontánea, rememorando sus primeros pasos en la } \\
\text { carrera; todas ellas sensaciones que se potencian en la presencialidad. } \\
\text { En PPDI: El establecimiento de vínculos afectivos entre pares, con docentes, con la asignatura, con la } \\
\text { carrera y con la institución. Considero que esto es una particularidad de los grupos de primer año. Si bien } \\
\text { cada año los docentes nos encontramos con nuevos grupos de estudiantes y la generación de vínculos } \\
\text { requiere de tiempo, en los estudiantes de años superiores hay vínculos preestablecidos (entre pares, con la } \\
\text { institución y hasta incluso con la asignatura, por lo que otros compañeros comentan o, en espacios corre- } \\
\text { lativos como son los espacios de PPD porque ya transitaron el primero) que sostienen el establecimiento } \\
\text { de nuevos vínculos. Con estudiantes de primer año, esta podría ser una primera gran limitación. }\end{array}$ \\
\hline P2 & $\begin{array}{l}\text { Imposibilidad de trabajo con libros de texto de editoriales variadas. Imposibilidad de vivenciar la simu- } \\
\text { lación de la planificación de una clase. Imposibilidad de efectivizar el trabajo en terreno. }\end{array}$ \\
\hline P3 & Creo que una limitación puede ser el trabajo en terreno propio del segundo semestre. \\
\hline
\end{tabular}


Al respecto, se coincide con Edelstein (2015) en que los espacios de la práctica están orientados a "favorecer la incorporación de los estudiantes a escenarios profesionales reales para vivenciar la complejidad del trabajo docente y, con relación al mismo, recuperar los saberes y conocimientos incorporados a lo largo del trayecto formativo a la vez que favorecer su profundización e integración" (p.4). El desafío aquí es cómo conjugarlo con una pedagogía de la virtualización en contexto de emergencia.

Al mismo tiempo visionan algunas posibilidades que emergen en el contexto virtual (Tabla 6), tanto para las docentes formadoras como para los/as estudiantes en su condición de practicantes:

- Volver a pensar acciones que, por diversos motivos, se presentaban como instaladas

- Ser claras y coherentes en la comunicación con el estudiantado

- Potenciar colaboración y coordinación

- Planificar con mayor detenimiento situándose en contextos específicos

- Emplear materiales alternativos para el trabajo en terreno

- Valorar este tipo de trabajo para posibles prácticas futuras

Tabla 6 | Posibilidades que emergen en el contexto virtual

\begin{tabular}{|c|c|}
\hline P & \multicolumn{1}{c|}{ Respuestas } \\
\hline P1 & $\begin{array}{l}\text { Desde el lugar de docentes emergen posibilidades de repensar acciones, formatos, modalidades de } \\
\text { trabajo, de acompañamiento, de intercambio de experiencias con otros espacios, de potenciar la gene- } \\
\text { ración de vínculos desde la virtualidad. Se potencia la concertación, la necesidad de establecer acuerdos } \\
\text { para poder transmitir claridad y coherencia a los estudiantes (y evitar así, una cuota más de incertidum- } \\
\text { bre o desconcierto). } \\
\text { En cuanto a los estudiantes, emergen posibilidades de potenciar el compañerismo, la colaboración, } \\
\text { el trabajo coordinado. }\end{array}$ \\
\hline P2 & $\begin{array}{l}\text { Al no poder efectuar la simulación de clases, en la planificación solicitaremos que, atendiendo a la pan- } \\
\text { demia, cada grupo diseñe actividades y explicaciones para la construcción/elaboración de un concepto, } \\
\text { de manera no presencial, para estudiantes de escasos recursos. Estimamos que será un desafío muy en- } \\
\text { riquecedor. Se les proporcionarán materiales (cuadernillos) que fueron elaborados por los Ministerios de } \\
\text { Educación de Nación y Pcia. de Santa Fe para los mismos fines. En cuanto al trabajo en terreno estamos } \\
\text { pensando en reemplazar las observaciones por el análisis de relatos de observación de estudiantes del año } \\
\text { 2019, actividad que creemos que atenderá a la mayoría de los objetivos del trabajo en terreno (claro que } \\
\text { nada reemplazará las vivencias...) y probablemente cubra otros. }\end{array}$ \\
\hline P3 & $\begin{array}{l}\text { El formar parte de este tipo de educación virtual nutre a los estudiantes con otras formas, herramientas, } \\
\text { modos de llevar a adelante estos procesos de enseñanza y de aprendizaje, que luego podrán valorarlas en } \\
\text { prácticas futuras. }\end{array}$ \\
\hline
\end{tabular}

Este tipo de trabajo, montado sobre formatos no presenciales, ha hecho palpable diversas facetas del conocimiento matemático para la enseñanza (Ball, Thames y Phelps, 2008) que cada estudiante va configurando como profesor/a en Matemática. Es en este sentido que la 
formación inicial "genera los cimientos de la acción profesional" (Davini, 2015, p.11), mediante la activación de dispositivos pensados, adaptados y re-diseñados para ello.

Adicionalmente, emergen otras consideraciones (Tabla 7) que las docentes desean compartir. P2 manifiesta cierta preocupación por la situación tanto actual (falta de contención) como en un futuro (retorno a clases) en un panorama incierto (Reimers, 2020) y P3 propone que ambas formas de trabajo (presencial-virtual) se combinen conveniente para sostenerse mutuamente, acorde a un enfoque mixturado (Bartolomé, 2004) que pueda asumir distintos matices según las circunstancias.

Tabla 7 | Otras consideraciones

\begin{tabular}{|c|c|}
\hline $\mathbf{P}$ & \multicolumn{1}{c|}{ Respuestas } \\
\hline $\mathbf{P 1}$ & $\begin{array}{c}\text { La mayor dificultad se presentará en el segundo cuatrimestre, con relación al trabajo en terreno. Aunque se } \\
\text { reabran las escuelas secundarias estas seguramente no estarán dispuestas a recibir practicantes para observar } \\
\text { (lo digo con conocimiento porque lo que están atravesando las escuelas, sus docentes y directivos es caótico). } \\
\text { Cuando regrese la actividad presencial habrá que restituir un sinnúmero de cuestiones relativas a los afectos y } \\
\text { la vida escolar en general. No sabemos si el retorno va a ser para "dar clase", es muy probable que no, al menos } \\
\text { durante un tiempo. En la misma línea, la verdad es que no sé cómo va a ser el regreso a las aulas de la FCEIA } \\
\text { en el segundo cuatrimestre (si es que se da). En el ámbito universitario también habrá que tomarlo con calma } \\
\text { y primero "sanar las heridas" de este tiempo que nos tomó por sorpresa y con la guardia baja. Siento que en la } \\
\text { situación que se está viviendo está faltando contención, tanto para docentes como para estudiantes. El presen- } \\
\text { te y el futuro se perciben como inciertos; intentamos sostener un vínculo educativo, con algunos aprendizajes, } \\
\text { como si nada estuviese sucediendo y eso lo veo preocupante. }\end{array}$ \\
\hline \multirow{2}{*}{ P3 } & $\begin{array}{l}\text { Creo que combinar las diferentes modalidades (virtuales y presenciales) desde la planificación de la } \\
\text { materia, hace que puedas sostenerte con algunas de ellas cuando la otra no esté, o al menos no resulte } \\
\text { ajeno su implementación. }\end{array}$ \\
\hline
\end{tabular}

\section{CONCLUSIONES}

Los principales asuntos a seguir trabajando pueden resumirse en dispositivos factibles que estén a la altura de conjugar sustanciosa y armoniosamente los desarrollos en Didáctica de la Matemática y en Tecnología Educativa, con un ojo ilustrado (Eisner, 1998) que pueda mirar de cerca y atender a críticas educativas constructivas en la Matemática Escolar. Desplegamos algunas posibilidades:

- Practicantes que realizan su trabajo en terreno tanto presencialmente en instituciones educativas como en entornos no presenciales.

- Tareas intelectualmente exigentes (González, 2000) en términos de Tecnología Educativa aplicada a la Matemática en los niveles educativos de desempeño.

- Profesionales que trabajen colaborativamente tanto para optimizar esfuerzos al servicio de una sociedad ávida en tiempo presente de una Educación Matemática de calidad. 
- Uso didáctico amplio de las tecnologías a disposición tanto en térmi nos didácticos como comunicacionales con el foco en favorecer habilidades matemáticas.

- Invención de propuestas didácticas diversas, flexibles e inclusivas que contemplen la realidad social de los/as estudiantes promovien do el acceso, permanencia y egreso en distintos niveles educativos.

La Educación Superior se ve desafiada en estos tiempos de pandemia e incertidumbre, en particular la Formación Docente en Matemática. Si bien se pretende que los espacios de las PPD sean transformadores, que se constituyan en lugares de producción de conocimiento original, de reflexión crítica sobre las prácticas educativas, donde volver a pensar la importancia de la contextualización de los contenidos y el codiseño de las clases, en las actuales circunstancias esencialmente conforman lugares donde las propuestas de enseñanzas poderosas (Maggio, 2012) sirvan de contención a los/as estudiantes. Paradójicamente la pedagogía de la virtualización en contexto de emergencia nos viene a revelar la necesidad imperiosa de una Universidad con rostros, ya sea por medio de una pantalla, de un audiovisual, de un dispositivo, invitándonos a apostar una vez más por esa relación única, personal y colectiva, que se construye en cada clase.

Paradójicamente la pedagogía de la virtualización en contexto de emergencia nos viene a revelar la necesidad imperiosa de una Universidad con rostros, ya sea por medio de una pantalla, de un audiovisual, de un dispositivo, invitándonos a apostar una vez más por esa relación única, personal y colectiva, que se construye en cada clase. 


\section{BIBLIOGRAFIA}

Ander-Egg, E. (2003). “Métodos y Técnicas de Investigación Social IV. Técnicas para la recogida de datos e información". Buenos Aires, Argentina: Lumen.

Ball, D., Thames, M. \& Phelps, G. (2008). “Content Knowledge for Teaching. What Makes It Special?". Journal of Teacher Education, 59(5), 389-407.

Bartolomé, A. (2004). "Blended Learning. Conceptos básicos. Píxel-Bit". Revista de Medios y Educación, (23), 7-20.

Consejo Interuniversitario Nacional (2013). Propuesta de Estándares para la Acreditación de los Profesorados Universitarios en Ciencias Exactas y Naturales (Res.CIN856/13). Buenos Aires, Argentina: Autor.

Copertari, S. y Trottini, A.M. (2013). "Experiencia a distancia Universitarias sin distancia. Hacia una pedagogía de la virtualización". En S. Copertari y S. Morelli (Comp.) (2013). Experiencias Universitarias de Enseñanzas a Distancia: Praxis, visiones y horizontes (pp.53-81). Rosario, Argentina: Laborde.

Davini, M.C. (2015). “La formación en la práctica docente”. Buenos Aires, Argetina: Paidós.

Edelstein, G. (2015). “La enseñanza en la formación para la práctica”. Educación, Formación e Investigación, 1(1), 1-11.

Eisner, E. (1998). "El ojo ilustrado. Indagación cualitativa y mejora de la práctica educativa". Buenos Aires, Argentina: Paidós.

Gerlero, C.A. (2014). "Los sentidos del compromiso social universitario. Una aproximación a la construcción del estado del arte". Revista de la Escuela de Ciencias de la Educación, (9), 129-146.

González, F. (2000). “Los Nuevos Roles del Profesor de Matemática. Retos de la Formación de Docentes para el Siglo XXI". Paradigma, 21(1), 139-172.

Kemelmajer, C. (09/04/2020). “Educación en tiempos de pandemia: consejos de especialistas para enriquecer las aulas virtuales". CONICET. Recuperado de:https://www.conicet.gov.ar/educacion-en-tiempos-de-pandemia-consejos-de-especialistas-para-enriquecer-las-aulas-virtuales/

Mishra, P. y Koehler, M. (2006). "Technological pedagogical content knowledge: A framework for teacher knowledge". Teachers College Record, 108(6), 1017-1054.

Maggio, M. (2012). "Enriquecer la enseñanza. Los ambientes con alta disposición tecnológica como oportunidad". Buenos Aires, Argentina: Paidós. 
Reimers, F.M. (08/05/2020). "El COVID-19 y la educación del futuro". Proyecto Educar 2050. Recuperado de https://www.kaltura.com/index.php/extwidget/preview/partner_id/1633051/uiconf_id/42959361/entry_id/1_pn8nrtrb/ embed/iframe?

Sanjurjo, L. (2005). "Volver a empezar. En L. Sanjurjo y X. Rodríguez". Volver a pensar la clase. Las formas básicas de enseñar (pp.11-138). Rosario, Argentina: Homo Sapiens.

Sanjurjo, L. (2009). "Razones que fundamentan nuestra mirada acerca de la formación de las prácticas". En L. Sanjurjo (Coord.). Los dispositivos para la formación en las prácticas profesionales (pp.15-40). Rosario, Argentina: Homo Sapiens. 